\title{
What are effective phonological units in Cantonese spoken word planning?
}

\author{
Andus Wing-Kuen Wong and Hsuan-ChiH Chen \\ The Chinese University of Hong Kong, Hong Kong, China
}

\begin{abstract}
Two picture-word interference experiments were conducted to investigate the nature of effective phonological units in Cantonese spoken word production. The names of the pictures were Cantonese monosyllables with a consonant + vowel + consonant $(\mathrm{CVC})$ structure. Participants' picture-naming responses were faster when the target (e.g., "star"/sing1/) and the distractor shared the same CVC component (e.g., 城/sing4/, meaning "city"), the same CV component (e.g., 食/sik6/, “eat”), or the same VC component (e.g., 境/ging2/, “region”), as opposed to when they were unrelated, and the facilitation effects observed were comparable in size. Also, similar facilitation effects were obtained across the $\mathrm{CV}+$ tone-related and the $\mathrm{VC}+$ tone-related conditions, whereas no reliable effect was found in the $\mathrm{V}+$ tone-related condition. These results indicate that an effective phonological unit in spoken word planning is neither a syllable (without tone) nor a segmental unit, and that the possible candidates lie between the two, at least in Cantonese.
\end{abstract}

A widely accepted notion in the language production literature holds that at least two processes are involved in formulating an utterance: One is the retrieval of lexicalsemantic and lexical-syntactic information, and the other is the generation of a word's phonological form (Caramazza, 1997; Dell, 1986; Levelt, Roelofs, \& Meyer, 1999). With regard to the latter, the received view is that segments (i.e., phonemes) are important phonological units. For instance, according to the WEAVER ++ model that was proposed by Levelt and colleagues, the segments of the target morpheme are spelled out in a parallel fashion at the beginning of phonological encoding; meanwhile, a metrical frame (which specifies the number of syllables in the word and the stress pattern) is constructed. Then, the ordered set of segments are associated with the frame incrementally from left to right, and the output of the phonological encoding process is a phonological word that can be used to access and activate the syllabic gestural scores stored in the mental syllabary for initiating the articulation processes. Note that although the existing models of speech production differ considerably from each other in how activation flows across levels, the prominent role of segments is relatively indisputable (Caramazza, 1997; Dell, 1986; Rapp \& Goldrick, 2000).

However, past studies on speech production were mostly conducted on Western languages with alphabetic scripts (e.g., Dutch and English). It is not self-evident that the same conclusion can be generalized to other languages with distinct properties (H.-C. Chen, 1992, 2006). To verify the universality of the existing production theories, the present study adopted Cantonese as a test case for the following reasons. First, unlike many Western languages, Cantonese is a tone language in which tone is lexically distinctive. It is still not clear how the existing production models can incorporate the unique function of tone in tone languages (J. Y. Chen, 1999; J. Y. Chen, Chen, \& Dell, 2002; Wong \& Chen, 2008). Second, the traditional linguistic approach used in describing the phonology of various Chinese dialects is quite different from the ones used among many Western languages. For instance, it has long been suggested that a syllable (without tone) in Cantonese, and also in Mandarin, is generally composed of an onset and a rhyme unit (Bruche-Schulz, 1997; Chao, 1968; Li \& Thompson, 1981). An onset is the consonantal beginning of a syllable and is usually a single consonant. The rhyme is the rest of the syllable, which might contain both a vowel and a coda. Note that the onset and the rhyme have been considered to be the minimal contrastive units in Cantonese speech according to the traditional view (Bruche-Schulz, 1997), but their role in speech preparation has not been clarified. In contrast, the WEAVER + + model described above makes no such distinction at the phonological level.

The picture-word interference (PWI) task, in which participants perform picture naming and ignore a distractor accompanying each picture, has frequently been adopted to investigate the process of speech preparation. Past PWI research on Western languages has shown that both begin-related distractors (i.e., the target and the distractor share the same word-initial segments) and end-related distractors facilitated participants' naming latencies, relative to the unrelated distractors (Damian \& Martin, 1999; Meyer \& Schriefers, 1991). Given these results, Levelt et al. (1999) argued that the locus of the phonological ef-

H.-C.Chen, hcchen@psy.cuhk.edu.hk 
fects obtained in a PWI task was situated at the segmental retrieval stage, during which the segments were spelled out in a parallel fashion.

In a Cantonese PWI study (Wong \& Chen, 2008), significant priming effects were found not only when targets and distractors shared the same syllable (with or without tone), but also when they shared only the same rhyme, regardless of the tone. No reliable effects were obtained if targets and distractors shared only the same syllable onset, or the same syllable onset and the tone. These results indicate that phonological units that are smaller than a syllable are also important in Cantonese spoken word planning. However, the nature of such subsyllabic representations is still unclear, and there are two alternating views on this. One (the traditional view) suggests that a Cantonese syllable should be divided into an onset and a rhyme, whereas the other (WEAVER ++ ) assumes that all the segments within a syllable (including the initial consonant, the vowel, and the coda) are equally important.

In the present study, two Cantonese PWI experiments were conducted to verify the two proposals above by using two types of distractors that shared with the targets the same degree of segmental overlap and either the same or a different rhyme. If the traditional view is held, significant priming should be observed only in the rhyme-related condition. This is because in our previous PWI study, a significant facilitation was observed when targets and distractors shared the same rhyme, but not when they shared merely the onset (Wong \& Chen, 2008). Alternatively, the WEAVER ++ model would predict comparable priming effects across the two types of distractors, since their degree of segmental overlap with the target is identical. In addition, since the syllable (without tone) in Mandarin has been shown to be an important planning unit during phonological encoding (J. Y. Chen et al., 2002), a syllablerelated condition was also included in the present study to see whether it is also the case in Cantonese. If so, a more robust priming effect should be obtained in the syllablerelated condition than in the rhyme-related condition. Furthermore, the distractors and targets used in Experiment 2 also shared the same lexical tone, but this was not the case in Experiment 1. Such manipulation was implemented to investigate the role of tone in Cantonese spoken word planning.

\section{EXPERIMENT 1}

The names of the pictures employed in Experiment 1 were all Cantonese monosyllables with a CVC structure (e.g., 星 /sing1/, meaning "star"). Four distractor conditions were included - namely, syllable- (without tone) related (e.g., 城/sing4/), CV-related (i.e., distractors and targets shared the same initial consonant and vowel; e.g., 食 /sik6/), VC-related (i.e., distractors and targets shared the same vowel and coda; e.g., 境 /ging2/), and unrelated control (e.g., 閣 /gok3/). Of particular interest was whether a reliable facilitation would be observed in the CV-related condition. The traditional view on Cantonese phonology would predict a null effect in this condition, since it has been demonstrated that no reliable effects were observed if the target and the distractor shared only an identical onset (Wong \& Chen, 2008). Alternatively, the WEAVER ++ model would expect comparable facilitation effects across the CV-related and the VC-related conditions, since the distractors in these two conditions have the same degree of segmental overlap with the target. Furthermore, if the syllable (without tone) in Cantonese is an effective phonological unit, a more robust priming effect should be found in the syllable-related condition as opposed to the CVrelated and the VC-related conditions.

\section{Method}

Participants. Thirty undergraduates from the Chinese University of Hong Kong who were native Cantonese speakers participated in this experiment.

Materials and Design. Twenty pictures were adopted from $\mathrm{Wu}$ (2001), which were all black-on-white line drawings with naming agreements above $80 \%$. The distractors were all visually presented, were orthographically unrelated to the name of the accompanying picture, and were matched in stroke number and visual character frequency across conditions (Chinese Character Database: With Word Formations, 2003) (all $F \mathrm{~s}<1)$

During the experiment, each participant received two blocks with 40 trials in each. There were equal numbers of trials from each distractor condition, and each picture appeared twice in each block. The order in which participants received the blocks was counterbalanced across participants. The items within each block were presented in a randomized order, but the same picture would not be repeated in consecutive trials.

Apparatus. The pictures and the distractors were presented on a Flex Scan 17-in. monitor, with a Dell PIII computer running the DMDX program. The pictures were digitized and displayed at a size of approximately $7.5 \times 12 \mathrm{~cm}$. The distractors were shown in LiSung 36 font. Vocal responses were recorded via a microphone connected to the computer. The computer scheduled the presentation of the stimuli and recorded the onset of the responses to the nearest millisecond by a voice-onset relay.

Procedure. Participants were tested individually. Each participant was first familiarized with the pictures by viewing each picture for $2 \mathrm{sec}$ with its name appearing below it. A practice block was then administered in which each picture was presented once and the participant was asked to name the picture. Any response other than the expected one was corrected by the experimenter.

Before each trial block, participants were instructed to ignore the accompanying Chinese character, and to name the picture through the microphone as quickly and as accurately as possible. In each trial, a fixation cross was first presented at the center of the screen for $500 \mathrm{msec}$, followed by a blank screen for $500 \mathrm{msec}$. After that, the target picture and the distractor were presented for $200 \mathrm{msec}$; then the distractor was replaced by a mask. The picture and the mask disappeared together once a vocal response was detected. The participant's naming latency was measured from the time when the picture was presented. The next trial began after a 1,000-msec blank.

\section{Results}

The three following types of responses were excluded from data analysis: incorrect responses $(1.38 \%)$, trials in which the voice key malfunctioned or mistriggered $(1.13 \%)$, and response times (RTs) that were $2.5 S D$ s beyond either participant or item mean $(4.3 \%)$.

Paired $t$ tests were performed to compare each phonologically related condition with the unrelated control condition. Significant facilitation effects, relative to those in the control condition, were found in the syllable-related 
condition $\left[t_{1}(29)=4.27, p<.001 ; t_{2}(19)=4.09, p=\right.$ $.001]$, the CV-related condition $\left[t_{1}(29)=3.31, p=.003\right.$; $\left.t_{2}(19)=3.01, p=.007\right]$, and the VC-related condition $\left[t_{1}(29)=4.06, p<.001 ; t_{2}(19)=2.25, p=.036\right]$. However, no significant differences were observed among the three phonological conditions [all $t \mathrm{~s} \leq 1.6$, and $p \mathrm{~s} \geq .12$ ]. The mean naming latencies (by participants) in various conditions are shown in Table 1. A similar analysis was conducted on the error data, but no significant results were found (all $t \mathrm{~s}<1$ ).

\section{Discussion}

In Experiment 1, comparable facilitation effects were observed across the CVC-related, CV-related, and VCrelated conditions, indicating that the syllable (without tone) in Cantonese might not be a distinct and important processing unit, at least at the early stage of phonological encoding. More importantly, the comparable priming effects obtained in the CV-related and the VC-related conditions are in line with the WEAVER ++ model, indicating that the priming effects were mainly induced by the degree of segmental overlap between the distractor and the target, irrespective of whether they shared a similar rhyme component (see, e.g., Schiller, 1998, for a similar segmental overlap account). Furthermore, the present results suggest that a significant facilitation would be observed, given that the distractor shared the same vowel plus an identical consonant with the target. Hence, if the lexical tone is represented or processed like a consonant unit, as was suggested by some previous speech error and aphasic patient studies (see Wong \& Chen, 2008, for a review), a reliable facilitation should also be observed if the distractor shared the same vowel and an identical tone with the target. Experiment 2 was thus conducted to examine this prediction and to investigate how tone is processed in relation to different segmental components.

\section{EXPERIMENT 2}

The names of the pictures adopted in Experiment 2 were also Cantonese monosyllables with a CVC structure (e.g., /sing1/). Four distractor conditions were includednamely, CVT-related (i.e., distractors and targets shared the same initial consonant, vowel, and tone; e.g., 式 /sik1/), VCT-related (i.e., distractors and targets shared the same vowel, coda, and tone; e.g., 京/ging1/), VT-related (i.e., distractors and targets shared the same vowel and tone, e.g., 必 / bit1/), and unrelated control. On the basis of the results of Experiment 1, significant priming effects would be expected in the CVT-related and the VCT-related

Table 1

Mean Naming Latencies (in Milliseconds), Standard Deviations, and Mean Error Rates (Err, in Percentage) Across Conditions in Experiment 1

\begin{tabular}{lccc}
\hline Condition & $M$ & $S D$ & Err \\
\hline CVC & 559 & 85 & 0.29 \\
CV & 563 & 72 & 0.25 \\
VC & 566 & 71 & 0.46 \\
Unrelated & 582 & 74 & 0.38
\end{tabular}

conditions. Comparing the magnitudes of these two facilitation effects can inform us about the relationship between lexical tones and segmental units. For instance, if tone is more closely associated with the word-final segments in the segmental-retrieval stage, a more robust facilitation should be observed in the VCT-related condition than in the CVT-related condition. Furthermore, a VT-related condition was included to see whether the lexical tone is processed in a manner similar to that for the segmental units in Cantonese. If so, a significant facilitation, which is comparable to the ones obtained in the CV-related and the VC-related conditions in Experiment 1, should also be observed in this condition.

\section{Method}

The method for this experiment was largely identical to that in Experiment 1 . Thirty fresh participants with the same characteristics as those in Experiment 1 participated for course credit. The stimuli were constructed using the same criteria as those in Experiment 1.

\section{Results}

The same criteria used for data trimming that were described in Experiment 1 were adopted. Overall, 1.46\% (incorrect responses), $1.63 \%$ (voice key mistriggered), and $4.1 \%$ (2.5 SD beyond either participant or item mean) of the original data were eliminated.

Paired $t$ tests were performed to compare each phonologically related condition with the unrelated control condition. Significant facilitation effects, relative to those in the control condition, were found in the CVT-related condition $\left[t_{1}(29)=2.14, p=.041 ; t_{2}(19)=2.15, p=.045\right]$ and in the VCT-related condition $\left[t_{1}(29)=2.49, p=.019\right.$; $\left.t_{2}(19)=3.17, p=.005\right]$, but no significant results were obtained for the VT-related condition $\left[t_{1}(29)=0.16, p=\right.$ $\left..87 ; t_{2}(19)=0.57, p=.58\right]$. Also, significant facilitation effects, relative to those in the VT-related condition, were observed in the CVT-related condition $\left[t_{1}(29)=3.25, p=\right.$ $\left..003 ; t_{2}(19)=2.11, p=.048\right]$ and in the VCT-related condition $\left[t_{1}(29)=3.68, p=.001 ; t_{2}(19)=2.66, p=.016\right]$. No other significant comparisons were found [all $t \mathrm{~s} \leq$ .42 , and $p s \geq .68]$. The mean naming latencies (by participants) in various conditions are shown in Table 2 . The error analyses revealed no significant results (all $t \mathrm{~s} \leq 1$ ).

\section{Discussion}

Comparable facilitation effects, relative to those of the control, were observed in the CVT-related and the VCTrelated conditions in Experiment 2, indicating that the tonal information is closely associated with neither the word-initial nor the word-final segments during the early stage of phonological encoding. Moreover, since signifi-

Table 2

Mean Naming Latencies (in Milliseconds), Standard Deviations, and Mean Error Rates (Err, in Percentage) Across Conditions in Experiment 2

\begin{tabular}{lccc}
\hline Condition & $M$ & $S D$ & Err \\
\hline CVT & 584 & 69 & 0.25 \\
VCT & 582 & 68 & 0.33 \\
VT & 601 & 72 & 0.46 \\
Unrelated & 599 & 74 & 0.42
\end{tabular}


cant priming effects were found in the CV-related and the VC-related conditions in Experiment 1, the null effect observed in the VT-related condition in Experiment 2 suggests that the lexical tone is probably represented or processed differently from segmental units. Furthermore, the significant difference observed between the VT-related condition and the remaining two phonologically related conditions in Experiment 2 echoes the findings of Experiment 1 . That is, a significant facilitation would be observed only when the distractor shared the same vowel, together with an identical consonant, with the target.

\section{GENERAL DISCUSSION}

In the present study, both of the beginning-related (e.g., CV-related or CVT-related) and the end-related (e.g., VC-related or VCT-related) distractors produced significant facilitation relative to those of the control, which replicated the typical findings in the literature (Meyer \& Schriefers, 1991; Schiller, 2008). This replication indicates that the position of overlap between the target and the distractor is irrelevant. However, the present results suggest that the degree of overlap between the target and the distractor matters (see Schiller, 1998). This is because no reliable effect was observed if the target and the distractor shared only a single segment, even together with the tone (e.g., VT-related condition). A significant facilitation effect was observed only when the target and the distractor shared the same vowel together with an identical consonant, but a second identical consonant would not enhance the facilitation effect to a significant extent. Furthermore, the null effect observed in the VT-related condition, together with the facilitation effects obtained in the CV-related and the VC-related conditions, indicates that the lexical tone is represented or processed differently from segmental units.

There is no clear evidence from the present study showing that the syllable (without tone) is an effective phonological unit, because otherwise a stronger facilitation should be observed in the CVC-related condition than in the CV-related and the VC-related conditions. This result seems in contrast to the findings of a Mandarin implicit priming study conducted by J. Y. Chen et al. (2002). In that study, reliable priming effects were obtained when the response words shared the same first syllable (with or without tone), but not when they shared only the same initial consonant or tone. Hence, they argued that the syllable (without tone) in Mandarin is an important planning unit at the phonological level. However, different paradigms might be sensitive to different processing stages. Indeed, it has been suggested that the implicit priming paradigm is more sensitive to the segment-to-frame association stage, whereas the PWI paradigm is more sensitive to the earlier, segmental-retrieval stage (Levelt et al., 1999). Accordingly, the present results suggest that the syllable (without tone) is not an important planning unit at the early stage of phonological encoding, at least in Cantonese.

Furthermore, the comparable facilitation effects observed between the CV-related and the VC-related condi- tions in Experiment 1, and those between the CVT-related and the VCT-related conditions in Experiment 2, suggest that a reliable facilitation would be observed, given that the target and the distractor shared the same vowel and an identical consonant regardless of whether they had the same rhyme component. Also, no reliable results were observed in our previous PWI study if the target and the distractor shared only an identical onset but had a different rhyme (Wong \& Chen, 2008). Together, these results are not consistent with the traditional view mentioned in the introduction, which assumes that the onset and the rhyme are the two fundamental processing units in Cantonese spoken word planning. Hence, the present results question the necessity for assuming distinct representations for such units as onsets and rhymes at the phonological level in Cantonese spoken word preparation.

Note, however, that the present findings are partly consistent with the WEAVER ++ model. For instance, our results indicate that the tone is not closely associated with the word-initial or the word-final segments, and that it is processed or represented differently from segmental units. These results can be accounted for by WEAVER ++ if one assumes that the tonal information is handled by a kind of frame unit similar to the metrical frame described in WEAVER + + (see J. Y. Chen et al., 2002, for a similar proposal). As was mentioned previously, WEAVER ++ proposes that a metrical frame that specifies the prosodic information of the target word is constructed independently from the segmental-retrieval process at the beginning of phonological encoding (Levelt et al., 1999). Consequently, differential patterns of results would be observed across segments and tones, as was demonstrated in the present PWI study.

Nevertheless, the null effect of the VT-related condition observed in the present study, and the null effects of the onset-related conditions reported in the previous Chinese word-production studies (J. Y. Chen et al., 2002; Wong \& Chen, 2008), do not favor the notion that individual segments are important in spoken word preparation. The results from these Chinese studies stand in marked contrast to the related research on Western languages in which reliable priming effects were obtained even when the prime shared only a single segment with the target (Roelofs, 1999; Schiller, 2008). Such discrepant results might be due in part to the effect of orthographic experiences. Dissimilar to many Western languages, Chinese adopts a logographic writing system in which each orthographically distinct unit (character) maps directly onto a syllable, but not a phoneme, in its spoken form. Indeed, there is evidence indicating that orthographic experience affects one's phonological awareness (see, e.g., Cheung, Chen, Lai, Wong, \& Hills, 2001). Consequently, the Chinese speakers might be less sensitive than the speakers of languages with alphabetic scripts to the similarity between the target and the distractor when they share only a single segment.

To conclude, the present findings suggest that on the one hand, the syllable (without tone) unit and the subsyllabic structures (such as onsets and rhymes) are not distinctively represented at the phonological level, and that, 
on the other hand, an individual segment is not sufficient to be an effective phonological unit in Cantonese spoken word planning. It appears that an effective phonological unit in Cantonese lies between a syllable and a segmental unit. Given these findings, some additional constraints regarding the assumptions of the phonological units (e.g., salience of individual segments) need to be considered by the existing models of speech production to fully account for the present results. Hence, the present study demonstrated the importance of cross-language research in developing a model of spoken word production with precision and universality.

\section{AUTHOR NOTE}

The present research was supported by General Research Fund Grant CUHK441008 from the Research Grants Council of the Hong Kong Special Administrative Region, China. We thank H. Cheung and M. Taft for helpful suggestions on the study and two anonymous reviewers for constructive comments on an earlier version of the manuscript. Correspondence concerning this article should be addressed to H.-C. Chen, Department of Psychology, The Chinese University of Hong Kong, Shatin, N.T., Hong Kong S.A.R., China (e-mail: hcchen@psy.cuhk.edu.hk).

\section{REFERENCES}

Bruche-Schulz, G. (1997). "Fuzzy" Chinese: The status of Cantonese in Hong Kong. Journal of Pragmatics, 27, 295-314.

Caramazza, A. (1997). How many levels of processing are there in lexical access? Cognitive Neuropsychology, 14, 177-208.

CHAO, Y. R. (1968). A grammar of spoken Chinese. Berkeley: University of California Press.

Chen, H.-C. (1992). Reading comprehension in Chinese: Implications from character reading times. In H.-C. Chen \& O. J. L. Tzeng (Eds.), Language processing in Chinese (pp. 175-205). Amsterdam: NorthHolland.

Chen, H.-C. (2006). Language processing: Implications from the study of Chinese. In Q. Jing, M. R. Rosenweig, G. d'Ydewalle, H. Zhang, H.-C. Chen, \& K. Zhang (Eds.), International progress in psychological science: Neural, cognitive, and developmental issues (pp. 367381). London: Psychology Press.

CHeN, J. Y. (1999). The representation and processing of tone in Man- darin Chinese: Evidence from slips of the tongue. Applied Psycholinguistics, 20, 289-301.

Chen, J. Y., Chen, T. M., \& Dell, G. S. (2002). Word-form encoding in Mandarin Chinese as assessed by the implicit priming task. Journal of Memory \& Language, 46, 751-781.

Cheung, H., Chen, H.-C., LaI, C. Y., Wong, O. C., \& Hills, M. (2001), The development of phonological awareness: Effects of spoken language experience and orthography. Cognition, 81, 227-241.

Chinese character database: With WORD Formations (2003) Retrieved on May 30, 2008, from http://humanum.arts.cuhk.edu.hk/ Lexis/lexi-can/.

Damian, M. F., \& Martin, R. C. (1999). Semantic and phonological codes interact in single word production. Journal of Experimental Psychology: Learning, Memory, \& Cognition, 25, 345-361.

Dell, G. S. (1986). A spreading-activation theory of retrieval in sentence production. Psychological Review, 93, 283-321.

Levelt, W. J. M., Roelofs, A., \& Meyer, A. S. (1999). A theory of lexical access in speech production. Behavioral \& Brain Sciences, 22, 1-75.

LI, C. N., \& Thompson, S. A. (1981). Mandarin Chinese. A functional reference grammar. Berkeley: University of California Press.

Meyer, A. S., \& Schriefers, H. (1991). Phonological facilitation in picture-word interference experiments: Effects of stimulus onset asynchrony and types of interfering stimuli. Journal of Experimental Psychology: Learning, Memory, \& Cognition, 17, 1146-1160.

RAPP, B., \& GoldRICK, M. (2000). Discreteness and interactivity in spoken word production. Psychological Review, 107, 460-499.

Roelofs, A. (1999). Phonological segments and features as planning units in speech production. Language \& Cognitive Processes, 14, 173-200.

SCHILLER, N. O. (1998). The effect of visually masked syllable primes on the naming latencies of words and pictures. Journal of Memory \& Language, 39, 484-507.

SCHILLER, N. O. (2008). The masked onset priming effect in picture naming. Cognition, 106, 952-962.

Wong, A. W.-K., \& Chen, H.-C. (2008). Processing segmental and prosodic information in Cantonese word production. Journal of Experimental Psychology: Learning, Memory, \& Cognition, 34, 11721190.

WU, S. Y. (2001). Modeling speech production: Implications from a Chinese study. Unpublished master's thesis, Chinese University of Hong Kong.

(Manuscript received December 15, 2008; revision accepted for publication June 4, 2009.) 